\title{
Quantum cascade laser-based tunable laser absorption spectroscopy for the detection of stable isotopes of $\mathrm{CO}_{2}$
}

\author{
P. Nitzsche ${ }^{1}$, C. Dinc ${ }^{1}$, K.Schmitt ${ }^{1}$ and J. Wöllenstein ${ }^{1,2}$ \\ ${ }^{1}$ Department of Microsystems Engineering (IMTEK), University of Freiburg, Georges-Köhler-Allee \\ 102, 79110 Freiburg, Germany \\ 2 Fraunhofer Institute for Physical Measurement Techniques (IPM), 79110 Freiburg, Germany \\ Tel.-Nr.: +49 761 203-96808, E-Mail: ponkanok.nitzsche@imtek.uni-freiburg.de
}

\begin{abstract}
We present a conceptual design and results on the determination of three stable carbon dioxide $\left(\mathrm{CO}_{2}\right)$ isotopologues. The isotopic analyses have been performed using a quantum cascade laser (QCL)based tunable laser absorption spectroscopy (TLAS) setup. A mid-infrared (MIR), tunable and narrow linewidth QCL is used as a light source to probe absorption lines at around $4.3 \mu \mathrm{m}$. Tuning the QCL in this selected region permits the simultaneous detection of three absorption lines of $\mathrm{CO}_{2}$ isotopologues including ${ }^{16} \mathrm{O}^{12} \mathrm{C}^{16} \mathrm{O},{ }^{16} \mathrm{O}^{13} \mathrm{C}^{16} \mathrm{O}$ and ${ }^{16} \mathrm{O}^{12} \mathrm{C}^{18} \mathrm{O}$. The result shows that the wavelength conversion method using the reference gas cell is well-suited for this technique.
\end{abstract}

Keywords: Gas Sensors, Optical Measuring System, Absorption Spectroscopy, Quantum Cascade Laser, Isotopic Composition Analysis.

\section{Motivation}

Measuring and monitoring of stable isotopic compositions of carbon dioxide $\left(\mathrm{CO}_{2}\right)$ play a crucial role in many research and industry fields such as in ecology [1], atmospheric sciences [2], breathe gas analysis [3] and combustion processes [4]. Since every photosynthesis and respiration processes of any biological systems in this planet influence unique changes in isotopic compositions, analysis of the stable isotopic fractionation of $\mathrm{CO}_{2}$ in atmosphere provides information about this biosphere-atmosphere exchange process. This could help us to understand more about the photosynthesis process and the discrimination against the heavier isotopes such as ${ }^{13} \mathrm{C}$ - or ${ }^{18} \mathrm{O}$-isotope by all plants [5].

Due mainly to anthropogenic activities including land use change, burning of biomass and fossil fuel, and modern industrial structure, large amount of greenhouse gases has been emitted into the atmosphere in last few decade [6]. This causes a shift in the balance of biogeochemical processes which are closely associated with an effect of global warming [7]. The study of stable isotope ratios of carbon in $\mathrm{CO}_{2}$ enable the investigation of the climate in the past and understanding the effects of the external forcing to the climatic system [8], thus allow an improvement of the climate model and prediction of future climate conditions [9], [10]. Moreover, studying of currently change of the natural isotopic ratio can help to identify and quantify sources and sinks for atmospheric trace gases [8].

In this contribution, an optical spectroscopic sensing technology based on the so-called tunable laser absorption spectroscopy (TLAS) [11] using a quantum cascade laser (QCL) as a light source is proposed in order to measure the isotopic ratio of atmospheric $\mathrm{CO}_{2}$. The underlying theoretical principle is presented. The criteria and methods considering for the line selection will be discussed. Based on the selected absorption lines the mid-infrared, tunable $\mathrm{QCL}$ around $4.3 \mu \mathrm{m}$ is chosen. The experimental setup for the detection of $\mathrm{CO}_{2}$-isotopic compositions is built and characterized. At last the detected signal and evaluated results are discussed.

\section{Theoretical background}

In convention, the expression of an isotopic signature or a ratio of stable isotopes is given in parts per thousand (per mil, \%o). The delta $\delta$ notation is often used to describe the isotopic abundance obtaining by comparison of isotope ratio of a sample ( $\left.R_{\text {sample }}\right)$ and a standard or reference material ( $\left.R_{\text {standard }}\right)$ [12]. As example, the abundance of ${ }^{13} \mathrm{C}$-isotope in $\mathrm{CO}_{2}$ gas yields as: 
$\delta^{13} \mathrm{C}\left(\mathrm{CO}_{2}\right)=\left(\frac{{ }^{13} R\left(\mathrm{CO}_{2}\right)_{\text {sample }}}{{ }^{13} R\left(\mathrm{CO}_{2}\right)_{\text {standard }}}-1\right) \cdot 1000 \%$

where

$$
\begin{aligned}
{ }^{13 \mathrm{C}} R\left(\mathrm{CO}_{2}\right) & =\frac{\left[{ }^{13} \mathrm{CO}_{2}\right]}{\left[{ }^{12} \mathrm{CO}_{2}\right]} \\
& =\frac{\text { abundance of rare isotope }}{\text { abundance of abundant isotope }}
\end{aligned}
$$

The reference material or isotopic standard is referred to VPBD-(Vienna Pee Dee Belemnite) scale. The standard value of ${ }^{13} \mathrm{C}$-isotopic ratio is given as ${ }^{13} R\left(\mathrm{CO}_{2}\right)_{\text {standard }(\mathrm{VPDB})}=0.01180$, as determined from the measurement of NBS 19 calcite [13].

Due to an individual structure and composition of each isotopologue of any molecules their absorption spectrum shows a specific profile. Since the variation of isotopic ratio in atmospheric surrounding is very small, midinfrared laser absorption spectroscopy is an appropriate technique used to determine the stable isotope abundances of atmospheric $\mathrm{CO}_{2}$. By ramping the injected laser current with a sawtooth or triangle signal, the laser's wavelength is probed over one or more absorption lines of interest, thus adequate absorption lines of $\mathrm{CO}_{2}$ isotopologues must be carefully selected. The transmitted laser intensity is detected with a photodetector after passing through the measuring gas as a signal. The Lambert-Beer law, Eq. (3) describes the relationship between the transmitted light, $I_{T}(\lambda)$ after propagating through the medium with an optical path length of $L$ and the material properties including the absorption coefficient, $\alpha(\lambda)$

$$
I_{T}(\lambda)=I_{0}(\lambda) \cdot e^{-\alpha(\lambda) \cdot L}
$$

Here, $\lambda$ denotes the wavelength and $I_{0}(\lambda)$ the initial light intensity at particular wavelength. Since the measurement sensitivity depends on the absorption path length, a long gas cell or a multi-pass cell can be used to improve the limit of detection of the setup. However, the wellknown limitation of this TLAS-technique is optical interference fringes rising from the Fabry-Pérot etalons between reflecting and scattering surfaces of the optical components [14].

\section{Lines selection}

Since we would like to spectroscopically measure the ratio of $\mathrm{CO}_{2}$-isotopologues, wellseparated absorption lines of each isotope must be selected. In the experimental design the selection of absorption lines detected by TLAS is typically based on two main criteria: (1) high absorption line strength and (2) a minimal interference with other gas lines in the measuring environment. In mid-infrared the absorption line strength of $\mathrm{CO}_{2}$ is almost two orders of magnitude larger than in near-infrared range, as illustrated in Figure 1. The abundance weighted line intensity of four $\mathrm{CO}_{2}$ isotopologues from $1.5 \mu \mathrm{m}$ to $5 \mu \mathrm{m}$ is simulated applying an appropriated line list from HITRAN2016-database [15] and the simulation is depicted in Figure 1.

In order to reach a sufficient absorption line strength, especially for the less abundant isotopologues, a mid-infrared light source in $4.3 \mu \mathrm{m}$ range is used in this TLASmeasurement. Moreover, there is sufficiently low interference with bother atmospheric gas spectra in this wavelength range, since the measurement should be performed in the atmospheric surrounding.

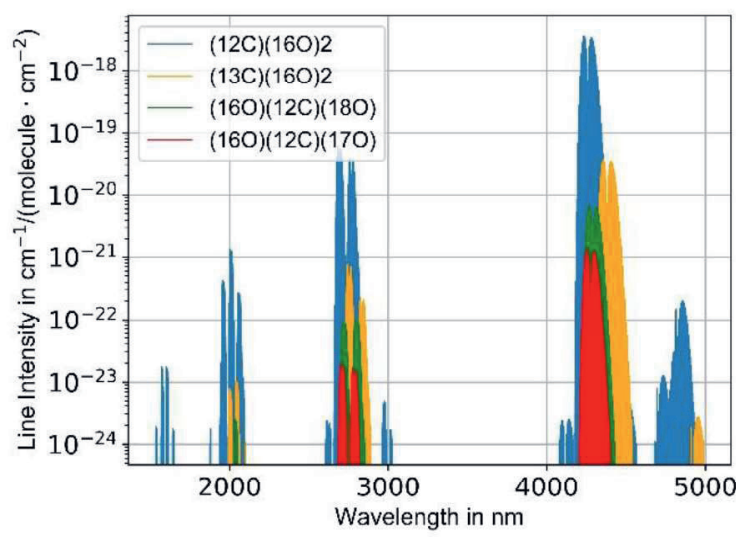

Fig. 2: The simulated line strength $S_{0}$ from $1.5 \mu \mathrm{m}$ to $5 \mu \mathrm{m}$ of four different isotopic compositions of $\mathrm{CO}_{2}$ using HITRAN2016-database [15]. Blue, yellow, green and red lines represent the weighted line strength of ${ }^{16} \mathrm{O}^{12} \mathrm{C}^{16} \mathrm{O}$, ${ }^{16} \mathrm{O}^{13} \mathrm{C}^{16} \mathrm{O},{ }^{16} \mathrm{O}^{12} \mathrm{C}^{18} \mathrm{O}$ and ${ }^{16} \mathrm{O}^{12} \mathrm{C}^{17} \mathrm{O}$, respectively.

However, further criteria must be considered when selecting the absorption lines for the spectroscopic detection of the isotopologues. In one tuning range of the laser's wavelength, there must be at least one absorption line for each isotopologue of interest so that one can simultaneously quantify their concentration and thus the ratio. As the weighted line strength of the most abundance isotope, Figure 2: blue line, is considerably higher than those with minority abundance, the absorption line of all measuring isotopologues should possess a similar line strength to avoid any detector saturation. 
A suitable tuning range have been found, in which the spectrum of at least three different $\mathrm{CO}_{2}$-isotopologues with comparable absorption line strength are located. The absorption spectra of four $\mathrm{CO}_{2}$-isotopologues from $4328.1 \mathrm{~nm}$ to $4329.3 \mathrm{~nm}$ with $407 \mathrm{ppm} \mathrm{CO}_{2}$ concentration which comprises $98.4204 \%$ ${ }^{16} \mathrm{O}^{12} \mathrm{C}^{16} \mathrm{O}$ (blue), $1.1057 \%{ }^{16} \mathrm{O}^{13} \mathrm{C}^{16} \mathrm{O}$ (yellow), $0.39471 \%{ }^{16} \mathrm{O}^{12} \mathrm{C}^{18} \mathrm{O}$ (green) and $0.0734 \%$ ${ }^{16} \mathrm{O}^{12} \mathrm{C}^{17} \mathrm{O}$ (red) are simulated and illustrated in Figure 2. According to HITRAN-database [15], this composition is referring to the natural abundance of $\mathrm{CO}_{2}$ isotope in atmosphere.

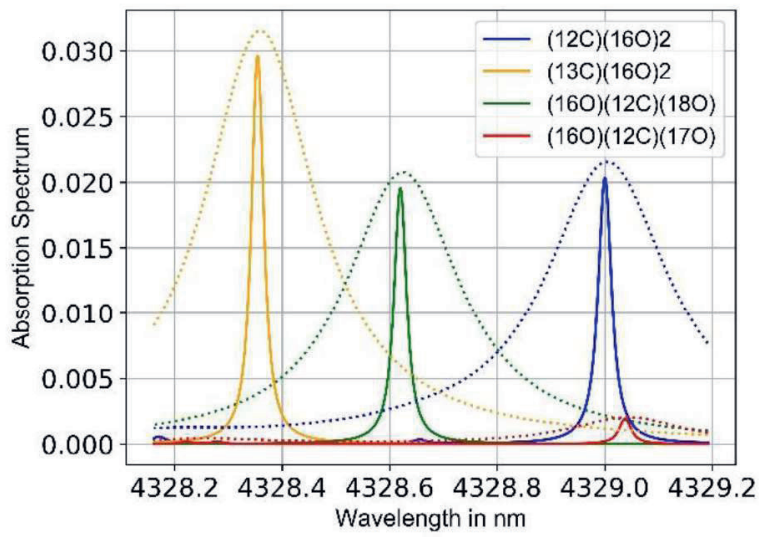

Fig. 2: The simulated absorption lines of four $\mathrm{CO}_{2}$-isotopologues from $4328.1 \mathrm{~nm}$ to $4329.3 \mathrm{~nm}$. The solid lines and dashed lines represent the simulation with gas pressure of 100 mbar and 1 bar, respectively [15].

The absorption path length is set to $100 \mathrm{~cm}$ and a temperature of $298 \mathrm{~K}$. The absorption spectra are simulated with two gas pressures 100 mbar and 1 bar represented by solid and dashed lines, respectively. To minimize problems caused by pressure broadening and $h$ the absorption line overlap, the measurement must be performed in a negative pressure chamber of 100 mbar. The absorption line of ${ }^{16} \mathrm{O}^{12} \mathrm{C}^{17} \mathrm{O}$ (red line) is however completely overlapped with the line of ${ }^{12} \mathrm{CO}_{2}$, thus the spectrum of ${ }^{16} \mathrm{O}^{12} \mathrm{C}^{17} \mathrm{O}$ in the measurement is not expected to be detected under this condition. In the selected spectral region, one can simultaneously measure three absorption lines with line center positions at $4328.354 \mathrm{~nm}$, $4328.619 \mathrm{~nm}$ and $4329.000 \mathrm{~nm}$ corresponding to isotopologues ${ }^{13} \mathrm{CO}_{2},{ }^{16} \mathrm{O}^{12} \mathrm{C}^{18} \mathrm{O}$ and ${ }^{12} \mathrm{CO}_{2}$, where ${ }^{13} \mathrm{CO}_{2}$ and ${ }^{12} \mathrm{CO}_{2}$ are ${ }^{13} \mathrm{C}^{16} \mathrm{O}_{2}$ and ${ }^{12} \mathrm{C}^{16} \mathrm{O}_{2}$, respectively.

\section{Experimental Setup}

Figure 3 (Top) depicts a scheme of experimental setup consisting of an optical system, control modules, signal acquisition module and gas flow systems. The image of the experimental setup is shown in Figure 3 (Bottom).
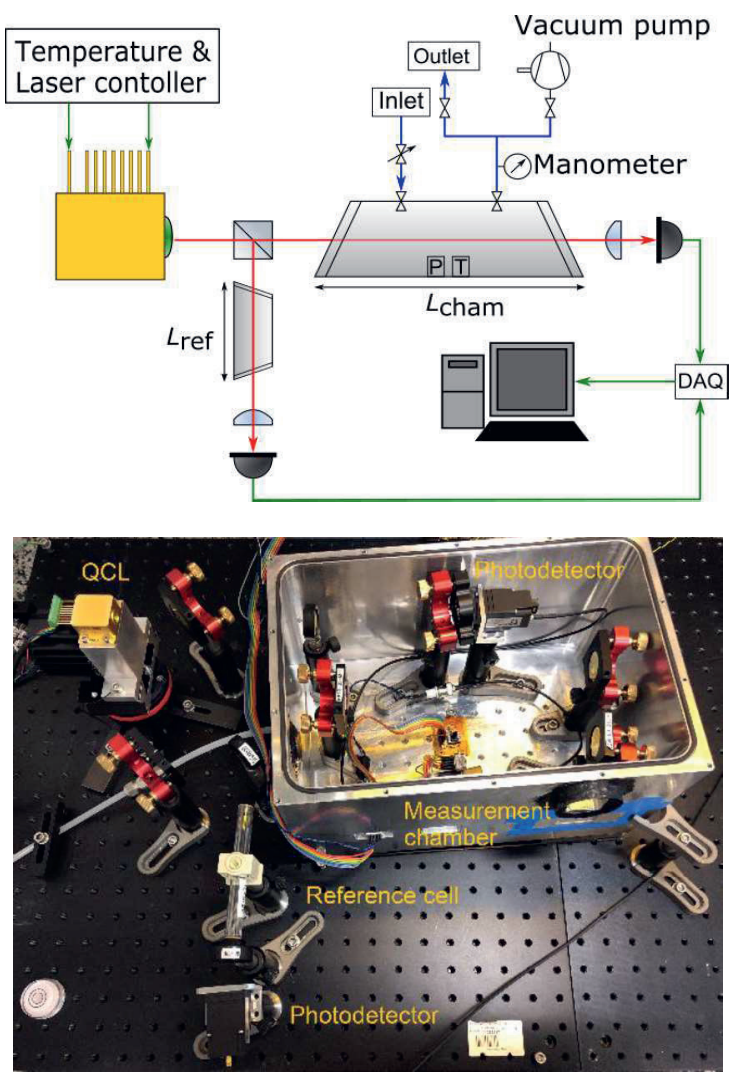

Fig. 3: Top: a schematic sketch of the experimental set-up for the QCLbased TLAS measurement. Bottom: photograph of the optical configuration of the setup.

The QCL (HHL680) from Alpes Lasers in HHLpackage with a collimation lens and a TECcooling unit is controlled by a laser driver (QCL1000LAB) and a temperature controller (PTC5K-CH) from Wavelength Electronics. Connecting a function generator (WaveStation2012) from LeCroy with the laser driver enables the laser's wavelength to be tuned around $4.32 \mu \mathrm{m}$ with a sawtooth signal and a ramp frequency of $f_{\text {ramp }}=1 \mathrm{~Hz}$. The beam splitter (BSW510) from Thorlabs splits the laser beam in two beams, where the first beam propagates through a hermetically sealed spectroscopic gas cell filled with $98.4 \%$ ${ }^{16} \mathrm{O}^{12} \mathrm{C}^{16} \mathrm{O}, 1.1 \%{ }^{16} \mathrm{O}^{13} \mathrm{C}^{16} \mathrm{O}$ and $0.5 \% \mathrm{~N}_{2}$ with a total pressure of $p_{\text {ref }}=10$ Torr or $13.332 \mathrm{mbar}$ which acts as a reference cell. The concentration of the filled gases and the condition in the reference cell are specified from the manufacturer, Wavelength References. The cell has an optical path length of $10 \mathrm{~cm}$. The reference signal is detected using a photodetector (PVI-3TE-5) from Vigo System 
with a neutral density filter from Thorlabs (NDIR20B).

The second beam propagates through the measured gas chamber, which tilted in two dimensions reducing the interference fringes. With three gold coated mirrors (PF10-03-M01) and a concave mirror (CM254-150-M01) with focal point of $150 \mathrm{~mm}$ from Thorlabs the laser beam is directed and reflected back and forth inside the chamber to obtain a total optical path of $L_{\text {cham }}=107 \pm 2.5 \mathrm{~cm}$. The absorption signal is likewise detected with Vigo-photodetector and a neutral density filter (NDIR30B). To reduce the gas pressure inside the chamber to $p_{\text {cham }} \approx 100$ mbar, a diaphragm vacuum pump (N840.3 FT.18) from KNF with a turn valve at the gas outlet and a fine-metering valve at the gas inlet are connected with the chamber to regulate the gas flow and pressure. Inside the measuring chamber a temperature sensor (SHT31-ARP-B) from Sensirion and pressure sensor (HSCMAND001BAAA5) from Honeywell are integrated. The detected signals including applied laser current and temperature, gas pressure and temperature in the chamber, photodetector signals and the modulation signal from the function generator are acquired using National Instrument DAQ cards (NI9205 and NI9239) with a sampling rate of $1 \mathrm{k}$ and $25 \mathrm{k}$ samples per second, respectively.

\section{Results and discussion}

According to the QCL's data sheet, the laser's temperature should be set to $-15^{\circ} \mathrm{C}$ in order to reach the selected tuning range from $4328.1 \mathrm{~nm}$ to $4329.3 \mathrm{~nm}$. In the experiment, we use the function generator to periodically change the laser's current at the chosen temperature. To ensure that the laser's wavelength alters in the desired range, we firstly observe the signal from the reference cell with an oscilloscope and compare it with the simulated spectrum to define the accordant voltage range for the sawtooth ramp. The frequency of the ramp is set to $1 \mathrm{~Hz}$.

The reference signal is used not only for the determination of the set-modulation voltage but also for the calibration of laser's emitted wavelength. However, the reference signal must be recorded synchronously with the TLASsignal so that the frequency calibration is likewise valid for the TLAS-signal. The reference signal as a function of counts is illustrated in Figure 4. The blue line is an averaged signal using a set of 20 time-series raw data corresponding to $20 \mathrm{~s}$. The red curve refers to the curve fit using a self-written algorithm in Python-program.

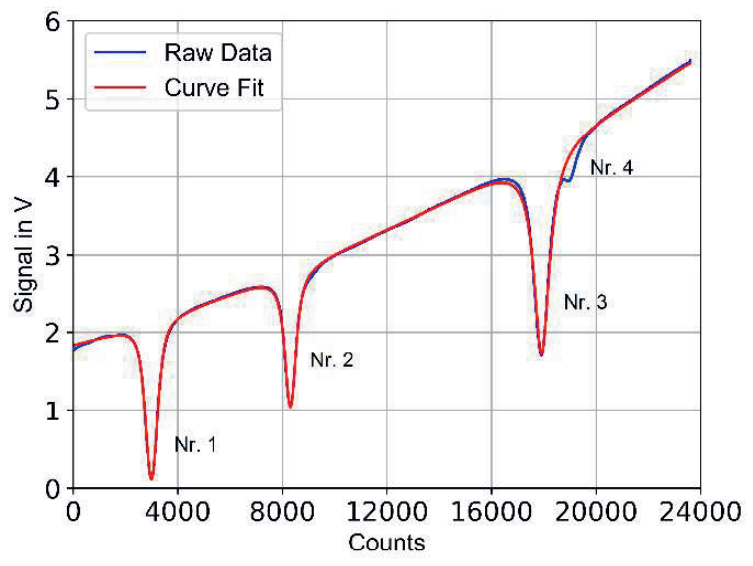

Fig. 4: The measured reference signal as a function of counts. The blue and red curves represent the average raw data and the curve-fit of it, respectively.

For the comparison, the reference signal has been simulated. The gas mixture inside the reference cell consisting of $98.4 \%{ }^{16} \mathrm{O}^{12} \mathrm{C}^{16} \mathrm{O}$, $1.1 \%{ }^{16} \mathrm{O}^{13} \mathrm{C}^{16} \mathrm{O}$, as told in the data sheet and the natural abundance for another two isotopologues is applied for the simulation. The simulated absorption spectrum is shown in Figure 5. The temperature is set to the averaged room temperature measured during the experiment $T=298.09 \pm 0.01 \mathrm{~K}$ and the pressure is set to $p_{\text {ref }}=10$ Torr.

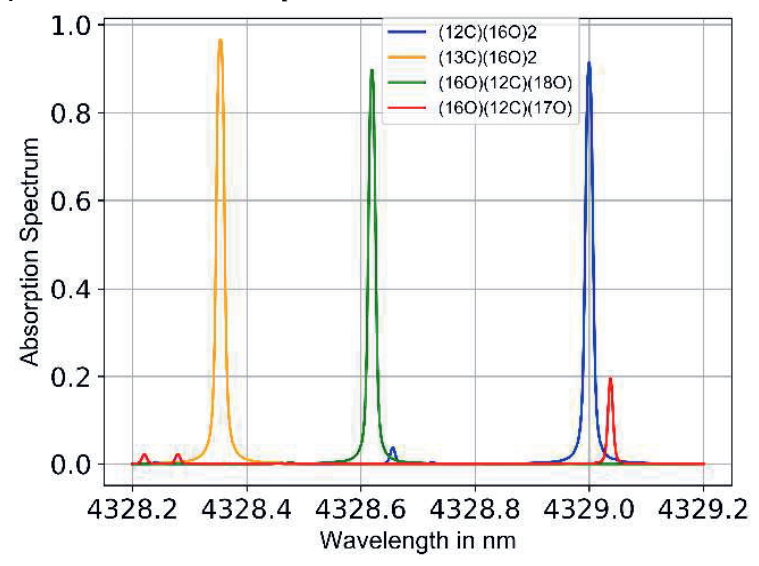

Fig. 5: The simulated reference signal of four isotopes using the HITRANdatabase [10]. The temperature is set to the averaged room temperature $T=298.09 \pm 0.01 \mathrm{~K}$.

Comparing the measured reference signal and those from the simulation, four absorption spectra of four different isotopologues can be identified. The spectrum of ${ }^{12} \mathrm{CO}_{2}$ and ${ }^{13} \mathrm{CO}_{2}$ masked as $\mathrm{Nr} .1$ and $\mathrm{Nr} .3$ can be clearly detected. The spectrum of ${ }^{16} \mathrm{O}^{12} \mathrm{C}^{18} \mathrm{O}(\mathrm{Nr} .2)$ and ${ }^{16} \mathrm{O}^{12} \mathrm{C}^{17} \mathrm{O}(\mathrm{Nr} .4)$ are not expected, however both spectra are detected in the measured signal. Even though the manufacturer can only confirm the filling of two isotopes with specific 
concentrations, using this spectroscopic method another isotope of $\mathrm{CO}_{2}$ in this reference gas cell can be identified.

Three center positions from the fitting parameters of the Nr.1 to Nr.3-spectrum have been taken for the calibration of the laser's wavelength. The center positions of the same spectra are likewise taken from the simulation in the unit of $\mathrm{nm}$. Employing these two sets of data, a conversion-function is calculated as:

$$
\lambda=a \cdot \text { counts }^{2}+b \cdot \text { counts }+c
$$

Here, $\lambda$ is the wavelength in $\mathrm{nm}$ and $a, b, c$ coefficients are $-7.0058 \times 10^{-13}, 5.7956 \times 10^{-5}$ and $4.3282 \times 10^{3}$, respectively. To gain a higher accuracy in frequency conversion a polynomial with higher degree can be used. For this application a quadratic polynomial is sufficient.

In order to measure the isotopes of atmospheric $\mathrm{CO}_{2}$, the pressure in the measuring chamber is reduced to about 100 mbar using the diaphragm vacuum pump. In this measurement, we use the laboratory-air as a test gas. The parallel TLAS-signal is detected with the sampling rate of $25 \mathrm{k}$ samples per second. To calculate the averaged signal, 20 periodical data-series are employed and the averaged signal against the number of counts is illustrated in Figure 6.

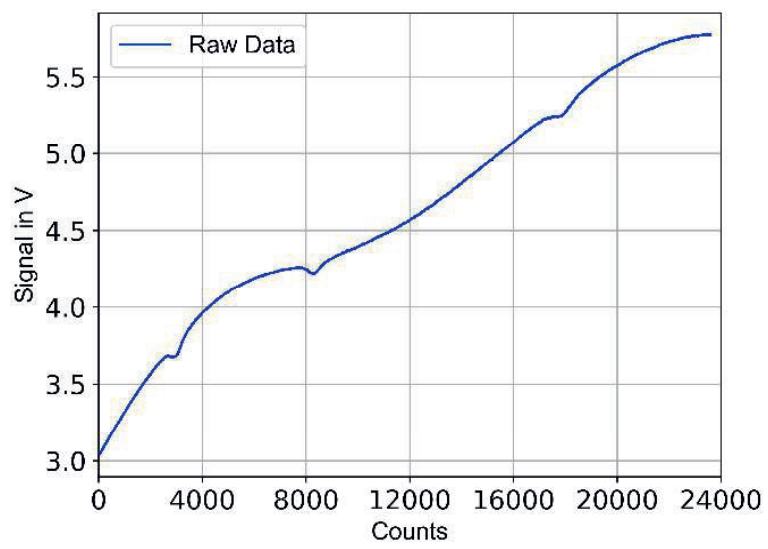

Fig. 6: The averaging of 20 data-series of TLAS-signal as a function of counts. The gas pressure and temperature in the measuring chamber is measured as $p_{\text {cham }}=99.5 \pm 0.3$ mbar and $T=298.09 \pm 0.01 \mathrm{~K}$.

The pressure and temperature inside the chamber is monitored using the integrated sensors during the measurement. The average values of the pressure and temperature over the $20 \mathrm{~s}$ is determined as $p_{\text {cham }}=99.5 \pm 0.3 \mathrm{mbar}$ and $T=298.09 \pm 0.01 \mathrm{~K}$, respectively.
The signal shows three absorption spectra of the isotopes as expected, however the baseline comprises strong interference fringes, which causing a difficulty in determination of the baseline. The signal around the position of absorption lines has been masked in order to determine a best-fit baseline function with a high-order polynomial function. Divided the averaged signal with this baseline function to remove the baseline from the signal, we obtained the transmittance. The negative natural logarithm of the transmittance, $-\ln \left(I / I_{0}\right)$ is calculated and shown in Figure 7 as a blue line. Employing the conversionfunction Eq. (5) to convert the counts number to wavelength, the transmittance can be plotted as a function of wavelength.

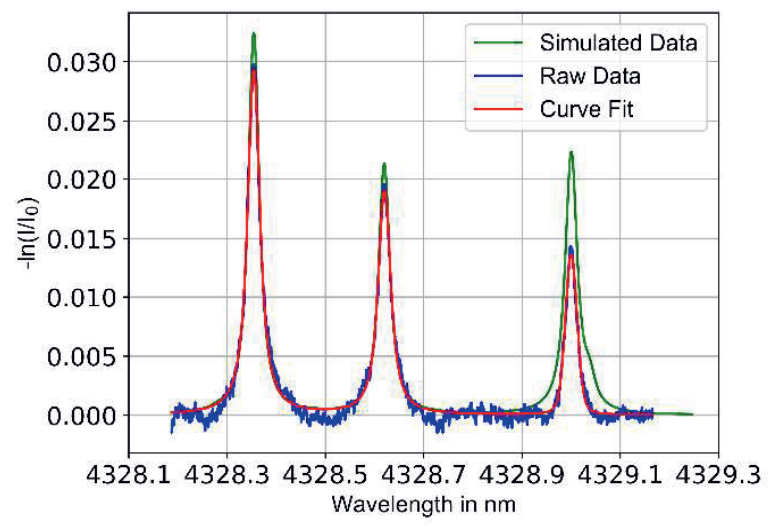

Fig. 7: The negative natural logarithm of the transmittance as a function of wavelength. Green, blue and red lines represent the simulated, raw data and the curve fit of the raw data.

A best fit-function of the transmittance spectrum has been computed using Voigt-profiles and illustrated as red line in Figure 7. The simulation of the negative logarithm of transmittance is likewise depicted as the green curve. The results show that the wavelength conversion method using information from the reference spectrum and simulation is sufficiently accurate for the calibration of the laser's frequency.

The first two spectra of the curve-fit corresponding to ${ }^{13} \mathrm{CO}_{2}$ and ${ }^{16} \mathrm{O}^{12} \mathrm{C}^{18} \mathrm{O}$ are in a well agreement with the simulation spectrum. However, the spectrum of ${ }^{12} \mathrm{CO}_{2}$ shows a deviation from the simulation. The calculated concentration obtained from this spectrum is thus not matching with the expected value, likewise the isotopic ratio. We suppose that the non-linearity of the detector could be a reason for this deviation. Further investigation of the system is required.

\section{Summary and outlook}

In this letter, the conceptual design and construction of an analysis of $\mathrm{CO}_{2}$-isotopic 
compositions using the so-called QCL-based tunable absorption spectroscopy have been presented. Three absorption lines of stable isotopologues, ${ }^{13} \mathrm{CO}_{2},{ }^{16} \mathrm{O}^{12} \mathrm{C}^{18} \mathrm{O}$ and ${ }^{12} \mathrm{CO}_{2}$ in $4.3 \mu \mathrm{m}$ range is selected. To reduce the pressure broadening effect, the measurement is performed in an under-pressure gas chamber. For the calibration of the laser's frequency, the reference gas cell is employed. The result confirms that the wavelength conversion for the TLAS-signal using the reference cell is wellmatched with the simulation.

However, further works must be undertaken. These include the calculation of the $\mathrm{CO}_{2}$ isotopic ratio, the evaluation of the long-term stability such as the thermal and pressure stabilities, and a measurement with a wellknown $\mathrm{CO}_{2}$-concentration and its isotopic compositions.

\section{Acknowledgements}

This work is partly funded by Georg H. Endress foundation.

\section{References}

[1] J. Balesdent, A. Mariotti, and B. Guillet, "Natural ${ }^{13} \mathrm{C}$ abundance as a tracer for studies of soil organic matter dynamics," Soil Biol. Biochem., vol. 19, no. 1, pp. 25-30, 1987.

[2] D. R. Bowling, S. D. Sargent, B. D. Tanner, and J. R. Ehleringer, "Tunable diode laser absorption spectroscopy for stable isotope studies of ecosystem-atmosphere $\mathrm{CO}_{2}$ exchange," Agric. For. Meteorol., vol. 118, no. 1-2, pp. 1-19, 2003.

[3] L. Cocola, A. Ghetti, G. Tondello, G. Galzerano, and L. Poletto, "Performance evaluation of a TDLAS system for carbon dioxide isotopic ratio measurement in human breath," Opt. Sens. Detect. V, no. May 2018, p. 31, 2018.

[4] M. Schumacher et al., "Oxygen isotopic signature of $\mathrm{CO}_{2}$ from combustion processes," Atmos. Chem. Phys., vol. 11, no. 4, pp. 14731490, 2011.

[5] D. R. Bowling, D. D. Baldocchi, and R. K. Monson, "Dynamics of isotopic exchange of carbon dioxide in a Tennessee deciduous forest," Global Biogeochem. Cycles, vol. 13, no. 4, pp. 903-922, 1999.

[6] IPCC, 2013: Summary for Policymakers. In: Climate Change 2013: The Physical Science Basis. Contribution of Working Group I to the Fifth Assessment Report of the Intergovernmental Panel on Climate Change [Stocker, T.F., D. Qin, G. K. Plattner, M. Tignor, S.K. Allen, J. Boschung, A. Nauels, Y. Xia, V. Bex and P.M. Midgley (Eds.)]. Cambridge University Press, Cambridge, United Kingdom and New York, NY, USA.

[7] E. A. G. Schuur et al., "Climate change and the permafrost carbon feedback," Nature, vol. 520, no. 7546, pp. 171-179, 2015.
[8] P. Ghosh and W. A. Brand, "Stable isotope ratio mass spectrometry in global climate change research," vol. 228, pp. 1-33, 2003.

[9] G. B. Bonan and S. C. Doney, "Climate, ecosystems, and planetary futures: The challenge to predict life in Earth system models," Science, vol. 359, no. 6375. 2018.

[10] A. Lourantou et al., "Constraint of the $\mathrm{CO}_{2}$ rise by new atmospheric carbon isotopic measurements during the last deglaciation," vol. 24 , no. C, pp. $1-15,2010$.

[11] D. D. Nelson, J. B. McManus, S. C. Herndon, M. S. Zahniser, B. Tuzson, and L. Emmenegger, "New method for isotopic ratio measurements of atmospheric carbon dioxide using a $4.3 \mu \mathrm{m}$ pulsed quantum cascade laser," Appl. Phys. B Lasers Opt., vol. 90, no. 2, pp. 301-309, 2008.

[12] W. A. Brand, T. B. Coplen, J. Vogl, M. Rosner, and T. Prohaska, "Assessment of international reference materials for isotope-ratio analysis (IUPAC Technical Report)," Pure Appl. Chem., vol. 86, no. 3, pp. 425-467, 2014.

[13] Q. Zhang and W. Li, "A calibrated measurement of the atomic weight of carbon," Chinese Sci. Bull, vol. 35, no. 4, pp. 290-296, 1990.

[14] J. Hodgkinson and R. P. Tatam, "Optical gas sensing: a review," Meas. Sci. Technol., vol. 24, no. 1, p. 12004, 2013.

[15] R. V. Kochanov, I. E. Gordon, L. S. Rothman, P. Wcisło, C. Hill, and J. S. Wilzewski, "HITRAN Application Programming Interface (HAPI): A comprehensive approach to working with spectroscopic data," J. Quant. Spectrosc. Radiat. Transf., vol. 177, pp. 15-30, 2016 